\title{
A Fuzzy Group Prioritization Method for Deriving Weights and its Software Implementation
}

\author{
Tarifa Almulhim, Ludmil Mikhailov and Dong-Ling Xu
}

Manchester Business School, University of Manchester, Booth Street East, Manchester

\begin{abstract}
Several Multi-Criteria Decision Making (MCDM) methods involve pairwise comparisons to obtain the preferences of decision makers (DMs). This paper proposes a fuzzy group prioritization method for deriving group priorities/weights from fuzzy pairwise comparison matrices. The proposed method extends the Fuzzy Preferences Programming Method (FPP) by considering the different importance weights of multiple DMs . The elements of the group pairwise comparison matrices are presented as fuzzy numbers rather than exact numerical values, in order to model the uncertainty and imprecision in the DMs' judgments. Unlike the known fuzzy prioritization techniques, the proposed method is able to derive crisp weights from incomplete and fuzzy set of comparison judgments and does not require additional aggregation procedures. A prototype of a decision tool is developed to assist DMs to implement the proposed method for solving fuzzy group prioritization problems in MATLAB. Detailed numerical examples are used to illustrate the proposed approach.
\end{abstract}

Keywords - Fuzzy Non-linear Programming, Fuzzy Preferences Programming Method, Multiple Criteria Decision Making, Triangular Fuzzy Numbers.

\section{INTRODUCTION}

$\mathrm{T}$ HERE are various techniques for deriving priorities/weights for decision elements (e.g. attributes/criteria) from a decision maker (DM) or group of DMs, some of which are reviewed by Choo and Wedley [1] and Ittersum et al. [2]. Most techniques are based on either direct weighting or on pairwise comparison. In direct weighting, the DM is directly asked to give values between 0 and 1 to each decision element to assign their importance. Some methods for deriving attributes/criteria weights by direct assigning techniques are: the Simple MultiAttribute Rating Technique (SMART) [3], SWING weighting methods [4], and SMART Exploiting Ranks (SMARTER) [5].

When the DM or the group of DMs are unable to directly assign decision elements' weights, the Pairwise Comparison (PC) method proposed in [6] can be used.

Psychological experiments have shown that weight derivation from $\mathrm{PC}$ is much more accurate than direct weighting [7]. Therefore, the PC methods are often used as an intermediate step in many MCDM methods, as Analytic Hierarchy Process (AHP) [7], Analytic Network Process (ANP) [8], PROMETHEE [9], and Evidential Reasoning (ER) [10].

The PC methods require construction of Pairwise Comparisons Judgment Matrices (PCJMs). In order to construct a PCJM, the DM is asked to compare pairwisely any two decision elements and provide a numerical/linguistic judgment for their relative importance. Thus, the DM gives a set of ratio judgments to indicate the strength of his/her preferences, which are structured in a reciprocal PCJM. Then, the weights or priority vectors of the decision elements can be derived from the PCJM by applying some prioritization methods.

There are numerous Pairwise Comparisons Prioritization Methods (PCPMs), such as the Eigenvector Method [7], the Direct Least Squares Method [11], the rank-ordering method [7], the Logarithmic Least Square Method [12], and the Fuzzy Programming Method [13]. Choo and Wedley [1] summarised and analysed 18 PCPMs for deriving a priority vector from PCJMs. They discussed that no method performs best in all situations and no method dominates the other methods.

However, in many practical cases, in the process of prioritization the DMs are unable to provide crisp values for comparison ratios. A natural way to deal with the uncertainty and imprecision in the DMs' judgments is to apply the fuzzy set theory [14] and to represent the uncertain DMs' judgments as fuzzy numbers. Thus, Fuzzy PCJMs can be constructed and used to derive the priority vectors by applying some Fuzzy PCPMs. Such methods are proposed by Laarhoven and Pedrycz's [15], Buckley [14], Chang [16] and Mikhailov [17], and applied for group decision making.

The existing fuzzy PCPMs have some drawbacks. They require an additional defuzzification procedure to convert fuzzy weights into crisp (non-fuzzy) weights. However, different defuzzification procedures will often give different solutions [17].

The linear and non-linear versions of the Fuzzy Preference Programming (FPP) method [17] do not require such defuzzification procedures, but their modifications for group decision making situations assume that all the DMs have the same weight of importance. However, in real group decision 
making problems, sometimes some experts are more experienced than others [18-19]. Therefore, the final results should be influenced by the degree of importance of each DM.

In order to overcome some of the limitations of the group FPP method, a new group version of the FPP method is proposed by introducing importance weights of DMs in order to derive weights for decision elements in group decision problems. The proposed method has some attractive features. It does not require any aggregation procedures. It does not require a defuzzification procedure. It derives crisp priorities/weights from an incomplete set of fuzzy judgments and incomplete fuzzy PCJMs. Moreover, the proposed method considers the DMs weights.

For applying the proposed method and solving prioritisation problems, a Non-Linear FPP Solver is developed based on the Optimization Toolbox of MATLAB, in order to overcome the complexity of programming. This decision tool is demonstrated by solving a few numerical examples.

The remainder of this paper is organised as follows. In Section II, representation of the fuzzy group prioritization problem is briefly explained. Then, the proposed method is presented in Section III and illustrated by numerical examples in section IV. The developed Non-Linear FPP Solver is presented in section $\mathrm{V}$, followed by conclusions.

\section{REPRESENTATION OF THE FUZZY GROUP PRIORITIZATION PROBLEM}

Consider a group of $K$ DMs $\left(D M_{k}, k=1,2, \ldots, K\right)$ that evaluate $n$ elements $\boldsymbol{E}_{\mathbf{1}}, \ldots \boldsymbol{E}_{\boldsymbol{n}}$ (in MCDM, these elements could be clusters, criteria, sub-criteria or alternatives). With respect to some fixed preference scales, each DM assesses the relative importance of any two elements $\left(E_{i}, E_{j}\right)(i, j=1,2, . ., n)$ by providing a ratio judgment $a_{i j k}$, specifying by how much $E_{i}$ is preferred/not preferred to $E_{j}$.

In a fuzzy environment, suppose that each DM provides a set of $y$ fuzzy comparison judgements $A^{k}=\left\{\tilde{a}_{i j k}\right\}$,

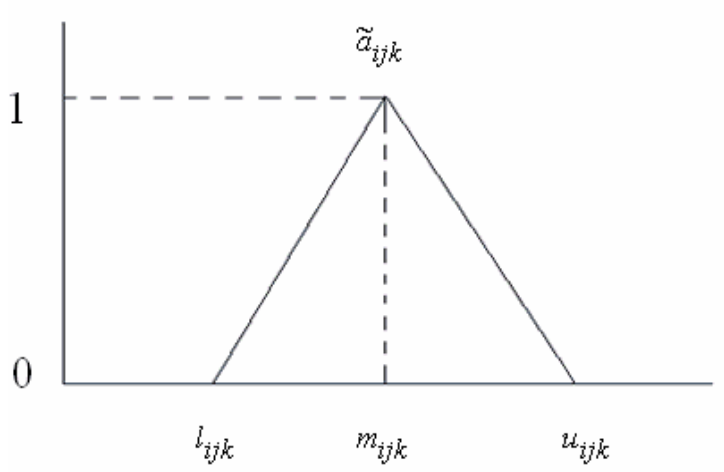

Fig. 1. Triangular Fuzzy Number $\tilde{a}_{i j k}=\left(l_{i j k}, m_{i j k}, u_{i j k}\right)$

$$
y \leq n(n-1) / 2, \quad \text { where } \quad i=1,2, . ., n-1, \quad j \succ i \quad,
$$$$
j=2,3, \ldots . n, k=1,2, . ., K \text { and those judgments are }
$$

represented as Triangular Fuzzy Numbers (TFNs) $\tilde{a}_{i j k}=\left(l_{i j k}, m_{i j k}, u_{i j k}\right)$, where $l_{i j k}, m_{i j k}$ and $u_{i j k}$ are the lower bound, the mode and the upper bound, respectively. Fig. 1 shows the TFN $\tilde{a}_{i j k}=\left(l_{i j k}, m_{i j k}, u_{i j k}\right)$.

The set $A^{k}$ can be used to form a Fuzzy PCJM of the form (1):

$A^{k}=\left[\begin{array}{cccc}(1,1,1) & \left(l_{12 k}, m_{12 k}, u_{12 k}\right) & \ldots & \left(l_{1 j k}, m_{1 j k}, u_{1 j k}\right) \\ \left(l_{21 k}, m_{21 k}, u_{21 k}\right) & (1,1,1) & \ldots & \left(l_{2 j k}, m_{2 j k}, u_{2 j k}\right) \\ \ldots & \ldots & \ldots & \ldots \\ \left(l_{i 1 k}, m_{i 1 k}, u_{i 1 k}\right) & \left(l_{i 2 k}, m_{i 2 k}, u_{i 2 k}\right) & \ldots & (1,1,1)\end{array}\right]$

Then, the fuzzy group prioritisation problem is to determine a crisp priority vector (crisp weights) $w=\left(w_{1}, w_{2}, \ldots, w_{n}\right)^{T}$ from all $A^{k}, k=1,2, \ldots, K$, which represents the relative importance of the $n$ elements.

\section{GROUP FuZZY PREFERENCE PROGRAMMING METHOD}

The non-linear FPP method [17] derives a priority vector $w=\left(w_{1}, w_{2}, \ldots, w_{n}\right)^{T}$, which satisfies:

$l_{i j} \tilde{\leq} w_{i} / w_{j} \tilde{\leq} u_{i j}$

where $\tilde{\leq}$ denotes 'fuzzy less or equal to'. If $M$ is the overall number of fuzzy group comparison judgments, then $2 M$ fuzzy constraints of the type (3) are obtained.

$$
\begin{aligned}
& -w_{i}+w_{j} l_{i j} \tilde{\leq} 0 \\
& w_{i}-w_{j} u_{i j} \widetilde{\leq} 0
\end{aligned}
$$

For each fuzzy judgment, a membership function, which represents the DMs' satisfaction with different crisp solution ratios, is introduced:

$$
\mu_{i j}\left(w_{i} / w_{j}\right)=\left\{\begin{array}{l}
\frac{\left(w_{i} / w_{j}\right)-l_{i j}}{m_{i j}-l_{i j}}, w_{i} / w_{j} \leq m_{i j} \\
\frac{u_{i j}-\left(w_{i} / w_{j}\right)}{u_{i j}-m_{i j}}, w_{i} / w_{j} \geq m_{i j}
\end{array}\right.
$$

The solution to the prioritization problem by the FPP method is based on two assumptions. The first, requires the existence of a non-empty fuzzy feasible area $\tilde{P}$ on the $(n-1)$ dimensional simplex $Q^{n-1}$,

$Q^{n-1}=\left\{\left(w_{1}, w_{2}, \ldots, w_{n}\right), w_{i} \succ 0, \sum_{i=1}^{n} w_{i}=1\right\}$ 
The fuzzy feasible area $\tilde{P}$ is defined as an intersection of the membership functions (4). The membership function of the fuzzy feasible area $\tilde{P}$ is given by:

$$
\mu_{\tilde{P}}(w)=\left[\operatorname{Min}\left\{\mu_{1}(w), \mu_{2}(w), \ldots . \mu_{2 M}(w)\right\} \backslash \sum_{i=1}^{n} w_{i}=1\right]
$$

The second assumption identifies a selection rule, which determines a priority vector, having the highest degree of membership in the aggregated membership function (6). Thus, there is a maximizing solution $w^{*}$ (a crisp priority vector) that has a maximum degree of membership $\lambda^{*}$ in $\tilde{P}$, such that :

$$
\lambda^{*}=\mu_{\tilde{P}}\left(w^{*}\right)=\operatorname{Max}\left[\operatorname{Min}\left\{\mu_{1}(w), \ldots \mu_{2 M}(w)\right) \backslash \sum_{i=1}^{n} w_{i}=1\right]
$$

A new decision variable $\lambda$ is introduced which measures the maximum degree of membership in the fuzzy feasible area $\tilde{P}$. Then, the optimization problem (7) is represented as

$\operatorname{Max} \lambda$

s.t.

$\lambda \leq \mu_{i j}(w)$

$\sum_{i=1}^{n} w_{i}=1, \quad w_{i} \succ 0$,

$i=1,2, \ldots, n, \quad j=1,2, . n, \quad j \succ i$

The above max-min optimization problem (8) is transformed into the following non-linear optimization problem:

$$
\begin{aligned}
& \operatorname{Max} \lambda \\
& \text { s.t. } \\
& \left(m_{i j}-l_{i j}\right) \lambda w_{j}-w_{i}+l_{i j} w_{j} \leq 0 \\
& \left(u_{i j}-m_{i j}\right) \lambda w_{j}+w_{i}-u_{i j} w_{j} \leq 0 \\
& i=1,2, . . n-1 ; j=2,3, . n ; j \succ i ; \\
& \sum_{i=1}^{n} w_{i}=1 ; \quad w_{i} \succ 0 ; \quad i=1,2, \ldots, n
\end{aligned}
$$

The non-linear FPP method can be extended for solving group prioritization problems. Mikhailov et al. [20] proposed a Weighted FPP method to the fuzzy group prioritization problem by introducing the importance weights of DMs. However, the Weighted FPP method requires an additional aggregation technique to obtain the priority vector at different $\alpha$ - thresholds. Consequently, this process is time consuming, due to several computation steps needed for applying the $\alpha$ threshold concept. Therefore, this paper modified the nonlinear FPP method [17], which can derive crisp weights without using $\alpha$ - threshold and by introducing the DMs' importance weights.

When we have a group of $K$ DMs, the problem is to derive a crisp priority vector, such that priority ratios $w_{i} / w_{j}$ are approximately within the scope of the initial fuzzy judgments $a_{i j k}$ provided by those DMs, i.e. $l_{i j k} \tilde{\leq} w_{i} / w_{j} \tilde{\leq} u_{i j k}$

The ratios $w_{i} / w_{j}$ can also express the satisfaction of the DMs, as the ratios explain how similar the crisp solutions are close to the initial judgments from the DMs.

The inequality (10) can be represented as two single-side fuzzy constraints of the type (3):

$R_{q}{ }^{k} W \widetilde{\leq} 0$,

$k=1, . ., K, q=1,2, .2 M_{k}$

The degree of the DMs' satisfaction can be measured by a membership function with respect to the unknown ratio $w_{i} / w_{j}$ :

$\mu_{q}{ }^{k}\left(R_{q}{ }^{k} W\right)=\left\{\begin{array}{l}\frac{\left(w_{i}^{k} / w_{j}^{k}\right)-l_{i j k}}{m_{i j k}-l_{i j k}}, w_{i}^{k} / w_{j}^{k} \leq m_{i j k} \\ \frac{u_{i j k}-\left(w_{i}^{k} / w_{j}^{k}\right)}{u_{i j k}-m_{i j k}}, w_{i}^{k} / w_{j}^{k} \geq m_{i j k}\end{array}\right.$

We can define $K$ fuzzy feasible areas, $\widetilde{P}_{k}$, as an intersection of the membership functions (12) corresponding to the $k$-th DMs' fuzzy judgments and define the group fuzzy feasible area $\widetilde{P}=\bigcap \widetilde{P}_{k}$.

By introducing a new decision variable $\lambda_{k}$, which measures the maximum degree of membership of a given priority vector in the fuzzy feasible area $\tilde{P}_{k}$, we can formulate a max-min optimisation problem of the type (8), which can be represented into:

$\operatorname{Max} \lambda_{k}$

s.t.

$\lambda_{k} \leq \mu_{q}{ }^{k}\left(R_{q}{ }^{k} W\right)$

$\sum_{i=1}^{n} w_{i}=1 ; \quad w_{i} \succ 0$,

$i=1,2, \ldots, n \quad k=1, \ldots, K ; \quad q=1,2, .2 M_{k}$

For introducing the DMs' importance weights, let us define $I_{k}$ as the importance weight of the $D M_{k} ; k=1,2, \ldots, K$. For aggregating all individual models of type (13) into a single group model, a weighted additive goal-programming (WAGP) model [21] is applied.

The WAGP model transforms the multi-objective decision making problem to a single objective problem. Therefore, it can be used to combine all individual models (13) into a new single model by taking into account the DMs' importance weights.

The WAGP model considers the different importance weights of goals and constraints and is formulated as: 
$\mu_{D}(x)=\sum_{s=1}^{p} \alpha_{s} \mu_{z_{s}}(x)+\sum_{r=1}^{h} \beta_{r} \mu_{g_{r}}(x)$

$\sum_{s=1}^{p} \alpha_{s}+\sum_{r=1}^{h} \beta_{r}=1$

Where:

$\mu_{z_{S}}$ are membership functions for the $p$-th fuzzy

goal $z_{S}, s=1,2, \ldots p$

$\mu_{g_{r}}$ are membership functions of the $h$-th fuzzy constraints

$g_{r}, r=1,2, . . h$;

$x$ is the vector of decision variables;

$\alpha_{s}$ are weighting coefficients that show the relative important of the fuzzy goals;

$\beta_{r}$ are weighting coefficients that show the relative important of the fuzzy constraints.

A single objective model in WAGP is the maximisation of the weighted sum of the membership functions $\mu_{z_{S}}$ and $\mu_{g_{r}}$. By introducing new decision variables $\lambda_{s}$ and $\gamma_{r}$, the model (14) can be transformed into a crisp single objective model, as follows:

$\operatorname{Max} \sum_{s=1}^{p} \alpha_{s} \lambda_{s}+\sum_{r=1}^{h} \beta_{r} \gamma_{r}$

s.t.

$\lambda_{s} \leq \mu_{z_{s}}(x), \quad s=1,2, \ldots p$

$\gamma_{r} \leq \mu_{g_{r}}(x), \quad r=1,2, . . h$

$\sum_{s=1}^{p} \alpha_{s}+\sum_{r=1}^{h} \beta_{r}=1$

$\lambda_{s}, \gamma_{r} \in[0,1] ; \quad \alpha_{s}, \beta_{r} \geq 0$

In order to derive a group model, where the DMs have different importance weights, we exploit the similarity between the models (13) and (15). However, the non-linear FPP model (13) does not deal with fuzzy goals; it just represents the nonlinear fuzzy constraints. Thus, by taking into account the specific form of $R_{q}{ }^{k} W \widetilde{\leq} 0$ and introducing the importance weights of the DMs, the problem can be further presented into a non-linear program by utilising the WAGP model as:

$$
\begin{aligned}
& \operatorname{Max} Z=\sum_{k=1}^{K} I_{k} \lambda_{k} \\
& \text { S.t. } \\
& \left(m_{i j k}-l_{i j k}\right) \lambda_{k} w_{j}-w_{i}+l_{i j k} w_{j} \leq 0 \\
& \left(u_{i j k}-m_{i j k}\right) \lambda_{k} w_{j}+w_{i}-u_{i j k} w_{j} \leq 0 \\
& i=1,2, . . n-1 ; j=2,3, . n ; \\
& j \succ i ; k=1,2, . K ; \\
& \sum_{i=1}^{n} w_{i}=1 ; \quad w_{i} \succ 0 ; \quad i=1,2, \ldots, n
\end{aligned}
$$

Where the decision variable $\lambda_{k}$ measures the degree of the
DM's satisfaction with the final priority vector $w=\left(w_{1}, w_{2}, \ldots \ldots . w_{n}\right)^{T} ; \quad I_{k}$ denotes the importance weight of the $k$-th DM, $k=1,2, . . K$.

In (16), the value of $Z$ can be considered as a consistency index, as it measures the overall consistency of the initial set of fuzzy judgments. When the set of fuzzy judgments is consistent, the optimal value of $Z$ is greater or equal to one. For the inconsistent fuzzy judgments, the maximum value of $Z$ takes a value less than one.

For solving the non-linear optimization problem (16), an appropriate numerical method should be employed. In this paper, the solution is obtained by using MATLAB Optimization Toolbox and a Non-linear FPP solver is developed to solve the prioritization problem.

\section{ILLUSTRATIVE EXAMPLES}

The first example illustrates the solution to the fuzzy group prioritization problem for obtaining a priority vector and a final group ranking. The second example demonstrates how the importance weights of DMs influence the final group ranking.

\section{A. Example 1}

This example is given to illustrate the proposed method and also the solution by using the Non-linear FPP Solver.

We consider the example in [20], where three DMs $(K=3)$ assess three elements $(n=3)$ and the importance weights of DMs are given as: $I_{1}=0.3 ; I_{2}=0.2 ; I_{3}=0.5$.

The DMs provide an incomplete set of five fuzzy judgments, presented as TFNs:

DM 1: $a_{121}=(1,2,3) ; a_{131}=(2,3,4)$.

DM 2: $a_{122}=(1.5,2.5,3.5) ; a_{132}=(3,4,5)$.

DM 3: $a_{123}=(2,3,4)$.

The group fuzzy prioritization problem is to derive a crisp priority vector $w=\left(w_{1}, w_{2}, w_{3}\right)^{T}$ that approximately satisfies the following fuzzy constraints:

For DM 1: $1 \tilde{\leq} w_{1} / w_{2} \tilde{\leq} 3 ; 2 \widetilde{\leq} w_{1} / w_{3} \tilde{\leq} 4$

For DM 2: $1.5 \widetilde{\leq} w_{1} / w_{2} \tilde{\leq} 3.5 ; 3 \widetilde{\leq} w_{1} / w_{3} \widetilde{\leq} 5$.

For DM 3: $2 \widetilde{\leq} w_{1} / w_{2} \tilde{\leq} 4$.

Using the above data and the non-linear model (16), the following formulation is obtained: 


$$
\begin{aligned}
& \operatorname{Max} Z=0.3 \lambda_{1}+0.2 \lambda_{2}+0.5 \lambda_{3} \\
& \text { s.t. } \\
& \lambda_{1} w_{2}-w_{1}+w_{2} \leq 0 \\
& \lambda_{1} w_{2}+w_{1}-3 w_{2} \leq 0 \\
& \lambda_{1} w_{3}-w_{1}+2 w_{3} \leq 0 \\
& \lambda_{1} w_{3}+w_{1}-4 w_{3} \leq 0 \\
& \lambda_{2} w_{2}-w_{1}+1.5 w_{2} \leq 0 \\
& \lambda_{2} w_{2}+w_{1}-3.5 w_{2} \leq 0 \\
& \lambda_{2} w_{3}-w_{1}+3 w_{3} \leq 0 \\
& \lambda_{2} w_{3}+w_{1}-5 w_{3} \leq 0 \\
& \lambda_{3} w_{2}-w_{1}+2 w_{2} \leq 0 \\
& \lambda_{3} w_{2}+w_{1}-4 w_{2} \leq 0 \\
& w_{1}+w_{2}+w_{3}=1 \\
& w_{1} \geq 0, \quad w_{2} \geq 0, \quad w_{3} \geq 0
\end{aligned}
$$

Regarding the judgments of this example, the results have been conducted by the Non-Linear FFP Solver. The solution to the non-linear problem (17) is:

$$
w_{1}=0.621, w_{2}=0.212, w_{3}=0.167 \text {. }
$$

This solution can be compared with the crisp results from the example in [20] as shown in Table I. We may observe that we have the same final ranking $w_{1} \succ w_{2} \succ w_{3}$, from applying the two different prioritization methods. However, the Weighted FPP method [20] applies an aggregation procedure for obtaining the crisp vector from different values of priorities at different $\alpha$-threshold. While, the proposed non-linear group FPP method does not require an additional aggregation procedure.

If the third DM, who has the highest important weight, provides a new fuzzy comparison judgment $a_{323}=(1,2,3)$, which means that the third element is about two times more important than the second element, the weights obtained by using the proposed Non-Linear FFP method are: $w_{1}=0.538, w_{2}=0.170, w_{3}=0.292$ and the final ranking is $w_{1} \succ w_{3} \succ w_{2}$. Consequently, it can be observed that the third DM's judgments strongly influence the final ranking.

However, if the importance weight of the third DM is lower TABLE I

RESULTS FROM THE TWO PRIORITIZATION METHODS

\begin{tabular}{cccc} 
Methods & $w_{1}$ & $w_{2}$ & $w_{3}$ \\
\hline Weighted FPP method $^{\mathrm{a}}$ & 0.615 & 0.205 & 0.179 \\
Non-linear FPP method $^{\mathrm{b}}$ & 0.623 & 0.216 & 0.161 \\
\hline \hline
\end{tabular}

${ }^{\mathrm{a}}$ The method proposed in [16] with applying $\alpha$-threshold.

${ }^{\mathrm{b}}$ The method proposed in this paper without applying $\alpha$ - threshold.

than the first two DMs' weights, then the new fuzzy comparison judgment does not change the final ranking. Thus, we can notice the significance of introducing importance weights of the DMs to the fuzzy group prioritization problem.

The computation time of the proposed method has been investigated by using the Non-Linear FFP Solver. It was found that the group non-linear FFP method performs significantly faster compared to the Weighted FPP [20] with different $\alpha$ thresholds ( $\alpha=0,0.2,0.5,0.8,1)$, as seen in Fig. 2 .

We can conclude that the average computation time (Minutes) for the Weighted FPP method highly increases as the number of decision elements $n$ increases, compared with the proposed method. Hence, these results showed that the method proposed in this paper is more efficient, with respect to the computation time. Therefore, the proposed method in this paper demands less computation time than the Weighted FPP method [20].

\section{B. Example 2}

This example shows that the importance weights of the DMs influence the final group ranking.

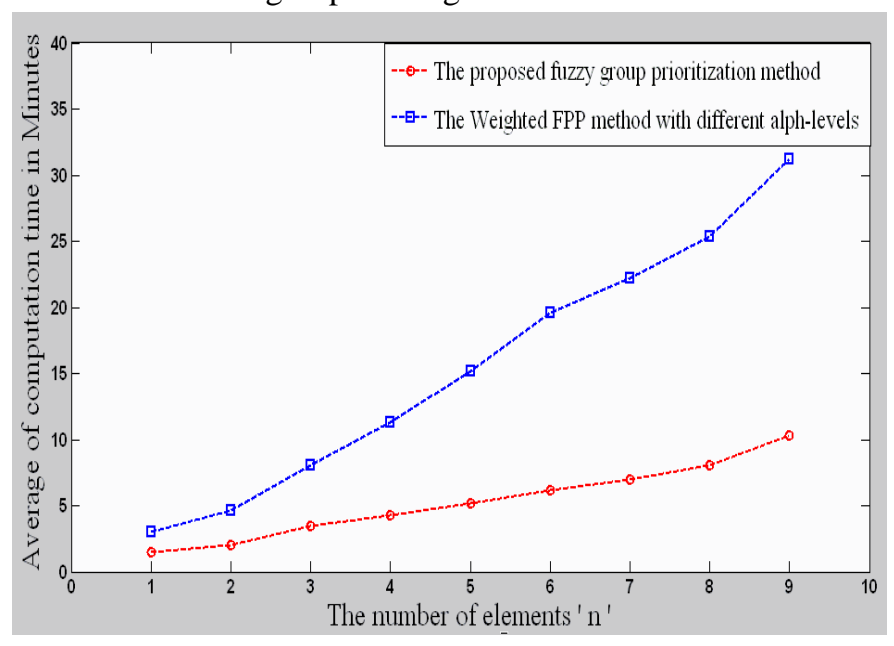

Fig. 2. Average Computation Time (Minutes)

Consider that two DMs ( $K=2$ ) assess three criteria $(n=3)$. The DMs provide an incomplete set of four fuzzy judgments $(m=4)$ presented as TFN:

DM 1: $a_{121}=(1,2,3) ; a_{131}=(2,3,4)$.

DM 2: $a_{212}=(3,4,5) ; a_{312}=(2,3,4)$.

Two situations are investigated when both DMs have the following different weights:

$$
\begin{aligned}
& \text { 1. } I_{1}=0.2, I_{2}=0.8 \\
& \text { 2. } I_{1}=0.8, I_{2}=0.2
\end{aligned}
$$

For both situations, the final rankings for both individual DMs are shown in Tables II and III respectively. The final group rankings are also shown in Tables II and III (the third row of each table). The results are obtained by using the Non-Linear FFP Solver. Each final group ranking is obtained by solving a non-linear program of type (15), which includes eight non-linear inequality constraints corresponding to the given DMs' fuzzy comparison judgements.

It can be observed from Tables II and III that the final group ranking tends to be the individual ranking of the 
DM who has the highest importance weights. In more detail, it can be seen from Table II that the judgements of the second DM with the highest importance weight $\left(I_{2}=0.8\right)$ influence, more strongly, the final group ranking. On the other hand, the final group ranking in Table III is dependent on the first DM, who has the highest importance weight $\left(I_{1}=0.8\right)$.

From examples 1 and 2 , we can observe the importance of introducing importance weights of the DMs to the fuzzy group prioritisation problem. It is seen that the final group ranking depends on the DMs' importance weights.

\begin{tabular}{|c|c|c|c|c|}
\hline \multicolumn{5}{|c|}{ V.SOFTWARE IMPLEMENTATION USING MATLAB } \\
\hline \multicolumn{5}{|c|}{$\begin{array}{r}\text { MATLAB is a numerical comp } \\
\text { TABLE II }\end{array}$} \\
\hline \multicolumn{5}{|c|}{ INDIVIDUAL AND GROUP RESULTS $\left(I_{1}=0.2, I_{2}=0.8\right)$} \\
\hline DMs & $w_{1}$ & $w_{2}$ & $w_{3}$ & Final ranking \\
\hline DM 1 & 0.545 & 0.273 & 0.182 & $w_{1} \succ w_{2} \succ w_{3}$ \\
\hline DM 2 & 0.117 & 0.530 & 0.353 & $w_{2} \succ w_{3} \succ w_{1}$ \\
\hline Group & 0.117 & 0.529 & 0.354 & $w_{2} \succ w_{3} \succ w_{1}$ \\
\hline
\end{tabular}

TABLE III

\begin{tabular}{ccccc}
\multicolumn{5}{c}{ INDIVIDUAL AND GROUP RESULTS $\left(I_{1}=0.8, I_{2}=0.2\right)$} \\
\hline \hline DMs & $w_{1}$ & $w_{2}$ & $w_{3}$ & Final ranking \\
\hline DM 1 & 0.545 & 0.272 & 0.181 & $w_{1} \succ w_{2} \succ w_{3}$ \\
DM 2 & 0.117 & 0.530 & 0.353 & $w_{2} \succ w_{3} \succ w_{1}$ \\
Group & 0.402 & 0.397 & 0.201 & $w_{1} \succ w_{2} \succ w_{3}$ \\
\hline \hline
\end{tabular}

allows matrix manipulations, plotting of functions and data, implementation of algorithms, creation of user interfaces, and interfacing with programs written in other languages, including C, C++, Java, etc. [22]. This development environment includes many functions for statistics, optimization, and numeric data integration and filtering [23].

In this paper, we use the Optimization Toolbox and the Graphical User Interface (GUI) of MATLAB as the development tools for implementing the proposed group nonlinear FPP method, because these tools provide powerful numerical functions, optimisation procedures, good visualisation capabilities and programming interfaces.

Essentially, there are three steps for programming and developing the Non-Linear FFP solver:

Step 1: Coding the model into the system. A number of functions are available in the Optimization Toolbox-MATLAB to solve the non-linear programming problem. In our prototype, the optimisation problem is solved using the sequential quadratic programming procedure [19].
Step 2: Creating a basic user interface. In this step, the interface is designed, so that it can run in the MATLAB command window. The aim of this user interface is to obtain the input information from the DMs.

Step 3: Developing the system based on the GUI functions. In this step, the MATLAB GUI functions are employed to develop a more user-friendly system.

Regarding the given data in example 1, the input information which should be acquired includes the total number of decision elements, the names of these elements and the total number of DMs, as shown in Fig.3. Then, the pairwise judgments for each DM can be entered by the user, as illustrated in Fig. 4. According to example 1, the fuzzy judgments for the DM 1 are illustrated in Fig. 4. Thus, the

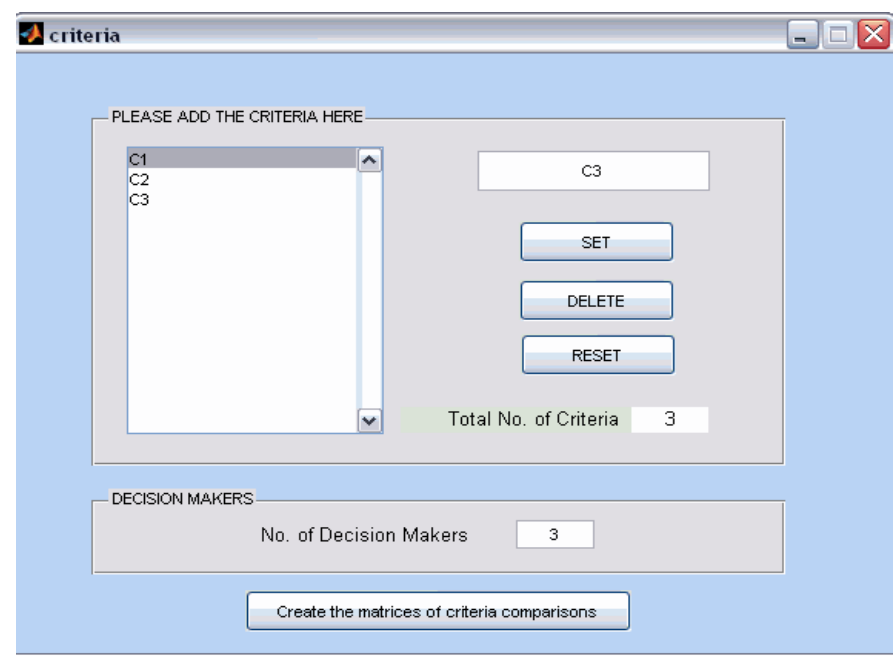

Fig. 3. The criteria setting window

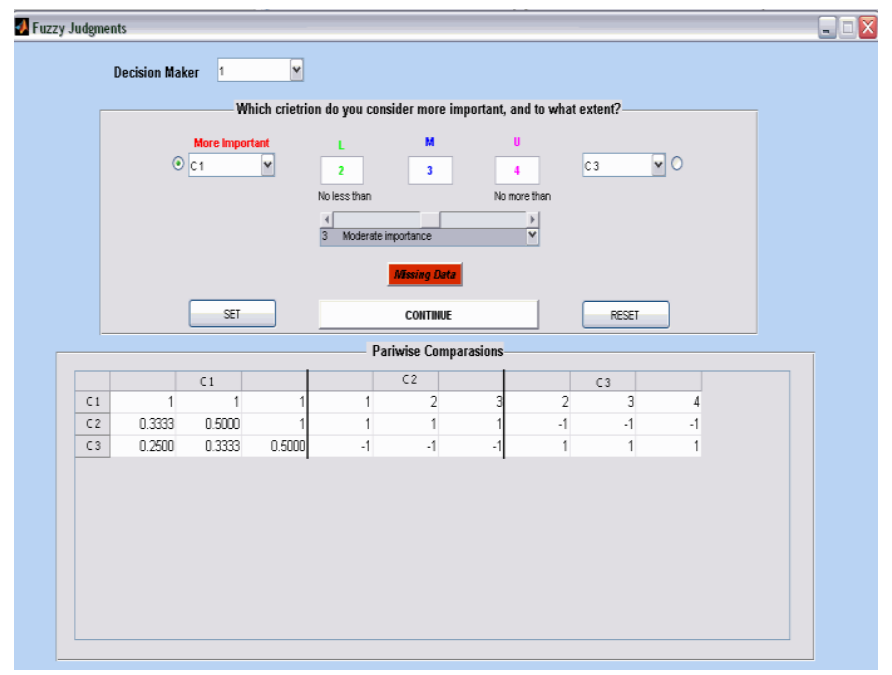

Fig. 4. The fuzzy comparison judgments window for the DM 1

main feature in the developed interface is that the user can input the fuzzy judgments into the system directly and easily.

However, if the user is unable to provide fuzzy comparison judgments between two elements, then he/she can click on the 'Missing Data' button and the system temporarily puts -1 for this comparison. The negative value is not a true judgment in the real world; it just indicates that those elements should 
not be included in the further calculations. For instance, in the given example, the judgment $a_{231}$ is missing for DM1 and it is recorded as $(-1,-1,-1)$ in Fig. 4.

After entering the fuzzy judgments from all DMs, the user can set the DMs' importance weights into the system. According to the given data in example 1, the importance weights of the three DMs are entered, as shown in Fig. 5.

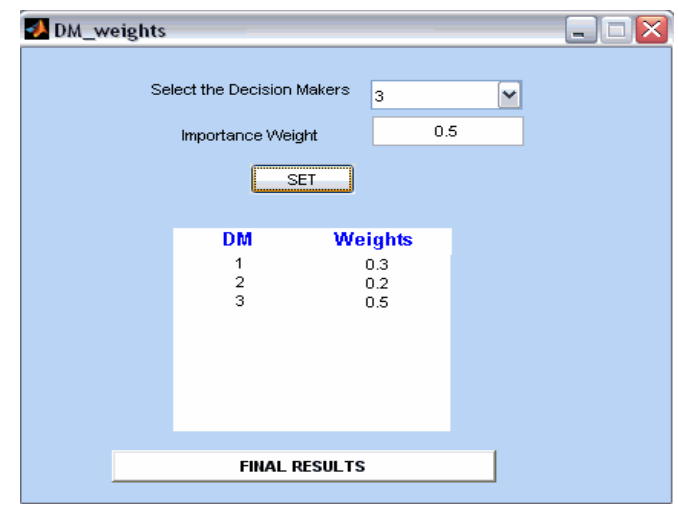

Fig. 5. The DMs' importance weights window

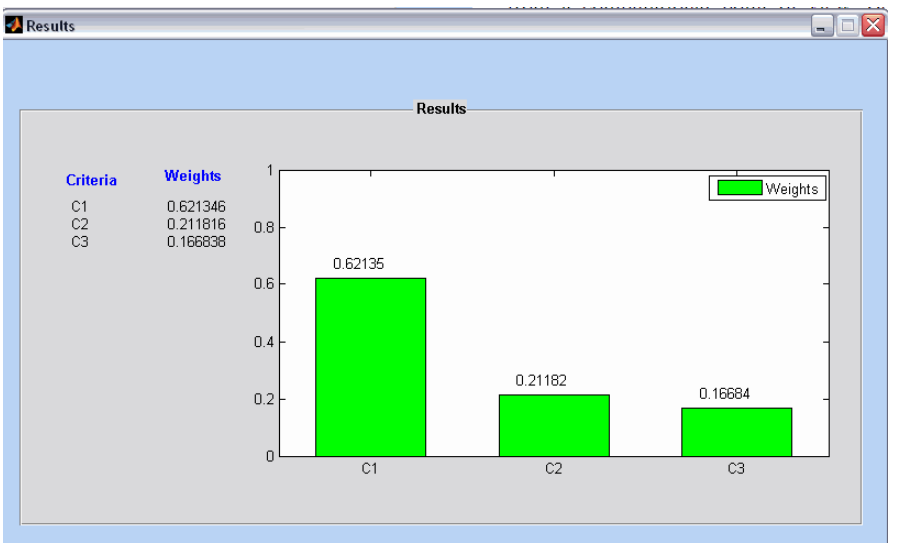

Fig. 6. The results from the Non-Linear FFP Solver

Finally, the Solver finds the optimal solution and visualises it graphically - Fig. 6.

\section{CONCLUSIONS}

This paper proposes a new method for solving fuzzy group prioritisation problems. The non-linear FPP is modified for group decision making by introducing DMs' importance weights. The proposed method derives crisp priorities/weights from a set of fuzzy judgements and it does not require defuzzification procedures. Moreover, the proposed method is capable of deriving crisp priorities from an incomplete set of DMs' fuzzy pairwise comparison judgments. Comparing with the Weighted FPP method, the proposed method is efficient from a computational point of view. Hence, the proposed method is a promising and attractive alternative method to existing fuzzy group prioritisation methods.

Another contribution of this study is the development of a Non-Linear FPP Solver for solving group prioritisation problems, which provides a user-friendly and efficient way to obtain the group priorities.

Future work includes presenting the importance weights for the DMs as fuzzy numbers, not just as crisp numbers, in order to model the uncertain importance weights of DMs. Moreover, we would like to incorporate the proposed method into other MCDM methods such as the Fuzzy Analytic Hierarchy Process, the Fuzzy Analytic Network Process and the Evidential Reasoning approach for complex decision problem analysis.

\section{REFERENCES}

[1] E. Choo, and W. Wedley, "A common framework for deriving preference values from pairwise comparison matrices," Computers \& Operations Research, vol. 31, no. 6, pp. 893-908, May 2004.

[2] K. V. Ittersum, J. M. Pennings, B. Wansink, and H. C. van Trijp, "The validity of attribute-importance measurement: A review," Journal of Business Research, vol. 60, no. 11, pp. 1177-1190, Nov. 2007.

[3] W. Edwards, "How to use multiattribute utility measurement for social decision making," IEEE Transactions on Systems, Man, and Cybernetics, vol. 7, no. 5, pp. 326-340, May 1977.

[4] D. V. Winterfeldt, and W. Edwards, Decision analysis and behavioural research. Cambridge, UK: Cambridge University Press, 1986.

[5] W. Edwards, and F. H. Barron, "SMARTS and SMARTER: Improved simple methods for multiattribute utility measurement," Organizational Behaviour and Human Decision Processes, vol. 60, no. 3, pp. 306-325, Dec. 1994.

[6] L. Thurstone, "A law of comparative judgment," Psychological review, vol. 101, no. 2, pp. 273-286, 1927.

[7] T. L. Saaty. The analytic hierarchy process: Planning, Priority setting, resource allocation. NY, McGraw-Hill, 1980.

[8] T. L. Saaty. Decision making with dependence and feedback: the Analytic Network Process. RWA Publications, PA: USA, 1996.

[9] J. Brans, B. Mareschal, "Promethee methods: In multiple criteria decision analysis: state of the art surveys," International Series in Operations Research \& Management Science, vol. 78, no. 2, pp. 163186, 2005.

[10] K. S. Chin, D. L. Xu, J. B. Yang, and J. Lama, "Group-based ER-AHP system for product project screening," Expert Systems with Applications, vol. 35, no. 4, pp. 1909-1929, Nov. 2008.

[11] A. Chu , R. Kalaba , and K. Springam, "A comparison of two methods for determining the weights of belonging to fuzzy sets," Journal of Optimization Theory and Application, vol. 27, no. 4, pp. 531-541, Apr. 1979.

[12] T. L. Saaty, L. G. Vargas, "Comparison of Eigenvalue, Logarithmic Least Squares and Least Squares Methods in Estimating Rations," Mathematical Modelling, vol. 5, no. 5, pp. 309-324, Mar. 1984.

[13] L. Mikhailov, "A Fuzzy Programming Method for Deriving Prioritises in Analytic Hierarchy Process," The Journal of Operational Research Society, vol. 51, no. 3, pp. 341-349, Mar. 2000.

[14] J. J. Buckley, "Fuzzy Hierarchical Analysis," Fuzzy Sets and System, vol. 17, no. 3, pp. 233-247, Dec. 1984.

[15] P. M. Laarhoven, and W. Pedrycz, "A fuzzy extension of Saaty's priority theory," Fuzzy Sets and System, vol. 11, no. 1, pp. 229-241, Feb. 1983.

[16] D. Chang, "Applications of the extent analysis method on fuzzy AHP," European Journal of Operational Research, vol. 95, no. 3, pp. 649655, Dec. 1996.

[17] L. Mikhailov, "Deriving priorities from fuzzy pair-wise comparison judgments," Fuzzy Sets and System, vol. 134, no. 3, pp. 365-385, Mar. 2003.

[18] R. Ramanathan, and L. S. Ganesh, "Group preference aggregation methods employed in AHP: An evaluation and an intrinsic process for deriving members' weightages," European Journal of Operational Research, vol. 79, no. 1, pp. 249-265, Dec. 1994.

[19] R.C. Van Den Honert, "Decisional power in group decision making: a note on the allocation of group members' weights in the multiplicative 
AHP and SMART," Group Decision and Negotiation, vol. 10, no.1, pp. 275-86, May 2001.

[20] L. Mikhailov, H. Didehkhani, and S. Sadi-Nezhad, "Weighted Prioritization Models in the Fuzzy Analytic Hierarchy Process," International Journal of Information Technology \& Decision Making, vol. 10, no. 4, pp. 681-694, Jul. 2011.

[21] R. N. Tiwari, S. Dharmar, and J. R. Rao, "Fuzzy goal programming-an additive model," Fuzzy Sets and System, vol. 24, no. 1, pp. 27-34, Oct. 1987.

[22] D. J. Higham, and N. J. Higham, MATLAB Guide. Second Edition, USA, PA, SIAM, 2005.

[23] J. G. Castellot, and J. L. Bello, "ADT-3D Tumor Detection Assistant in 3D," International Journal of Interactive Multimedia and Artificial Intelligence, vol.1, no.1, pp. 6-15, Dec. 2008.
Tarifa Almulhim received a bachelor degree in mathematics from King Faisal University, Alhassa, Saudi Arabia in 2005, and a M.Sc. degree in operational research and applied statistics from the University of Salford, Salford, UK, in 2010.

She has worked as a lecturer at King Faisal University and has tutored in various subjects, including Operational Research, Applied Statistics as well as the use of SPSS software on behalf of the Statistics and Quantitative Methods Department. Currently, she is working towards her Ph.D. in business and management at the University of Manchester, UK. Her current research interests include studying the health insurance market in developing countries, multi-criteria decision methods and analytical decision processes.

Ludmil Mikhailov obtained a first class BSc (1974) and MSc (1976) degree in Automatic Control from the Technical University in Sofia, Bulgaria and a $\mathrm{PhD}$ degree in Technical Cybernetics and Robotics from the Bulgarian Higher Certifying Commission (1981).

$\mathrm{He}$ is a Senior Lecturer at the Manchester Business School, the University of Manchester.

He worked as an Associated Professor at the Institute of Control and System Research of the Bulgarian Academy of Sciences (BAS) until 1996, where he was a head of a research group. During his work at BAS, he participated in many industrial projects on the development of various software systems for control, monitoring and technical diagnosis.

After joining the Decision Technologies Group at the Computation Department, UMIST in January 1997, Ludmil started to investigate new methods for multiple criteria decision making. He is the author of about 90 technical papers in peer-reviewed journals and international conferences, and holds two patents in the area of systems and control. His current research interests include multiple criteria decision analysis, fuzzy logic systems, decision making under uncertainty and intelligent decision support systems.

Dong-Ling Xu (PhD, MBA, MEng, BEng) received her BSc degree in electrical engineering, Master degree in Business Administration (MBA), and $\mathrm{MSc}$ and $\mathrm{PhD}$ degrees in system control engineering.

She is Professor of decision sciences and systems in Manchester Business School, the University of Manchester.

Prior to her current appointment, she worked as lecturer and associate professor in China, principal engineer in industry and research fellow in universities in the UK. She has published over 150 papers in journals and conferences, such as European Journal of Operational Research and IEEE Transactions on Systems, Man, and Cybernetics.

As a co-designer, she developed a Windows based decision support tool called IDS (Intelligent Decision Systems). The tool is now tested and used by researchers and decision analysts from over 50 countries, including organisations such as NASA, PricewaterhouseCoopers and General Motors. Her current research interests are in the areas of multiple criteria decision analysis under uncertainties, decision theory, utility theory, optimisation and their applications in performance assessment for decision making, including supplier selection, policy impact assessment, environmental impact assessment, sustainability management and consumer preference identification. 